\title{
Importance of optimal fiber consumption during
}

$$
\text { pregnancy }
$$

\author{
Laleh Hajhoseini* ${ }^{*}$
}

Article History:

Received July 2013

Accepted August 2013

Available online September 2013

\section{Keywords: \\ Fiber \\ Nutrition \\ Pregnancy}

\section{Corresponding Author:}

Laleh Hajhoseini, Department of Food and Nutrition Science, San Jose State University, CA, USA.

Tel:

Email: laleh_haj@yahoo.com

\section{Dear Editor}

Fiber has known as an essential part of human nutrition. It is a nutrient that could not get digested by human gastrointestinal tract. It absorbs water and helps bowel movements. Fiber Classifies as polysaccharides such as cellulose, pectin, lignin, etc. Two types of fiber exist, soluble and insoluble. Soluble fiber absorbs water; binds to fatty acids; slows down the rate of sugar absorption; helps stomach emptying and gets fermented via GI (gastrointestinal) bacteria through digestion. Insoluble fiber goes without changes through digestion; it helps to maintain the PH level of intestines and it also helps to move bulks through GI. Some types of soluble fibers are: kidney beans, pinto beans, brussels sprouts, broccoli, spinach, zucchini, fruits (raw), apple, orange, grapefruit, grapes, prunes, grains, oatmeal, whole-wheat bread. Some types of insoluble fiber are: vegetables (especially dark green leafy ones), root vegetable skins, fruit skins, whole wheat products, wheat bran, corn bran, nuts and seeds (14). The daily recommended intake of fiber is 25-35 gm for pregnancy; however, in many countries the consumed fiber is below $14 \mathrm{grm} /$ day for pregnant women. Adequate hydration and water intake is recommended along with proper fiber intake. Excess of fiber is not recommended because it causes nutrient loss and diarrhea during pregnancy.

Fiber is effective in prevention of many GI diseases and cancer (11). Fiber rich diet gives optimal calorie control and early satiety in weight loss plans. It is an important part of pregnancy diet both for mother and fetus health. Increase of fiber content in the diet during pregnancy should be gradual and start with soluble fiber to avoid bloating and flatulence. Moderate exercise along with fiber intake is recommended for pregnant women with the direct control of managing physician. Proper fiber intake avoids constipation and hemorrhoids during pregnancy (12).

Antioxidants balance related to fiber consumption has a provital role in development of fetus during pregnancy. It has shown that after consumption of fiber rich diet, the antioxidant capacity of SOD (superoxide dismutase) increases significantly but placental MDA (malondialdehyde) level decreases

1-Centro Escholar university, manila, Philippines. 
significantly in high fiber diet. Moreover, the antioxidants scavenging potential of free radicals get increased with fiber right diet. All these affect on normal fetal development and health. Oxidative stress is known to affect on pathogenesis of hypertension and preeclampsia during pregnancy so it is postulated that consumption of high fiber, fruits and vegetables rich in antioxidants could be beneficial in ameliorating hypertension, but further studies appear to be needed on this matter. Antioxidants could pass through placenta and selenium antioxidant has been shown to be affective in structure of GPX (glutathione peroxidase) enzyme, so it is related to decrease rate of miscarriage and preeclampsia $(1,7,10)$.

Plant lignans(nuts, seeds, legumes, fruits and vegetables) are fibers known with antioxidant virtue. They are associated with reduced risk of cardiovascular disease. Their phytochemicals likely to contribute to their health benefits. Lignans have antiestrogenic parts that mimic estrogen hormone but do not have the same antagonistic virtue and reduces risk of ovarian and uterine cancer rate. Breast cancer is another health problem which is avoided by phytoesterogens of lignans. There are studies indicating that lignans have improved effect in reproductive function. Besides, colon cancer prevention, hair growth and diabetes prevention are among lignans' benefits. Lignan's high antioxidant capacity make them a better immune system defender $(5,6)$.

Allergic developing disease in early childhood is a direct consequence of low fruits and vegetable consumption of a mother during pregnancy. Pregnant women who had higher consumption of fiber showed to have babies with low incidence of developing asthma(4). Eating whole grains in pregnancy would reduce risk of tube defects, spina bifida, and anencephaly during fetal development. Whole grains are also good source of magnesium and selenium. Magnesium is used to make bones and to release energy from muscles.
Selenium has antioxidant potential and protects cells from oxidation so it is a key factor in immune system (8). According to recent studies, whole grains are also full source of vitamin B which plays a crucial role in metabolism and nervous system. Folate (folic acid), another B vitamin, is essential in development of red blood cells during pregnancy. Furthermore, folate deficiency is linked to various complications including low birth weight (13).

Higher fiber consumption during the second trimester of pregnancy is associated with reduced insulin resistance in participants with family history of type 2 diabetes. Gestational diabetes, a common condition occurs in pregnancy, is wellcontrolled with fiber rich diet (15). There are reports of organic food consumption with high sources of vegetables and fruits which considered healthy during pregnancy. Those who consume strict vegetarian diet during pregnancy need to consult with their doctor and nutrition professionals to make sure the protein and vitamin supplementation along with vegetarian diet are provided $(3,16)$. A randomized trial study for Latino pregnant women has shown that dietary education for improving dietary behaviors and life style changes could improve fiber consumption in this population(2).

To summarize fiber is an important part of daily diet and it should be considered vital for pregnancy due to many benefits. It is required for both mother and fetus health. Fiber helps the proper digestion during pregnancy; provides important nutrients such as vitamin B groups; helps pregnancy weight gain and to be under control; helps optimal blood glucose control; prevents constipation; prevents cardiovascular disease during pregnancy and finally prevents later childhood allergy developments. To better include fiber in pregnant women diet recommend to consume fresh fruits in the breakfast, whole grain breads and cereal for breakfast; snacks and a lot of fresh or cooked vegetables for lunch and dinner. 


\section{References:}

1. Lin Y, Han XF, Fang ZF, Che LQ, Wu D, Wu XQ, Wu CM. The beneficial effect of fiber supplementation in high- or low-fat diets on fetal development and antioxidant defense capacity in the rat. 2012; 51(1):19-27.

2. Kieffer EC, Welmerink DB, Sinco BR, Welch KB, Rees Clayton EM, Schumann CY, Uhley VE. Dietary Outcomes in a Spanish-Language Randomized Controlled Diabetes Prevention Trial With Pregnant Latinas.Am J Public Health. 2013; Epub ahead of print]

3. Torjusen H, Lieblein G, Næs T, Haugen M, Meltzer HM, Brantsæter AL. Food patterns and dietary quality associated with organic food consumption during pregnancy; data from a large cohort of pregnant women in Norway. BMC Public Health. 2012; 12:612.

4. Peroni DG, Bonomo B, Casarotto S, Boner AL, Piacentini GL. How changes in nutrition have influenced the development of allergic diseases in childhood. Ital J Pediatr. 2012; 38:22.

5. Luoto R, Kharazmi E, Saarinen NM, Smeds AI, Mäkelä S, Fallah M, Raitanen J, HilakiviClarke L. Effect of dietary intervention on serum lignan levels in pregnant women - a controlled trial. Reprod Health. 2010; 7:26.

6. Carmichael SL, Gonzalez-Feliciano AG, Ma C, Shaw GM, Cogswell ME.Estimated dietary phytoestrogen intake and major food sources among women during the year before pregnancy. Nutr J. 2011; 10:105.

7. Watanabe K, Mori T, Iwasaki A, Kimura C, Matsushita H, Shinohara K, Wakatsuki A. Increased oxygen free radical production during pregnancy may impair vascular reactivity in preeclamptic women. Hypertens Res. 2013; 36(4):356-60.

8. Fenzl V, Flegar-Meštrić Z, Perkov S, Andrišić L, Tatzber F, Žarković N, Duić Ž. Trace elements and oxidative stress in hypertensive disorders of pregnancy.Arch Gynecol Obstet. 2013;287(1):19-24.

9. Mistry HD, Williams PJ. The importance of antioxidant micronutrients in pregnancy. Oxid Med Cell Longev. 2011; 841749.

10. Qiu C, Coughlin KB, Frederick IO, Sorensen TK, Williams MA. Dietary fiber intake in early pregnancy and risk of subsequent preeclampsia. Am J Hypertens. 2008; 21(8):903-9.

11.Yu B, Khan G, Foxworth A, Huang K, Hilakivi-Clarke L. Maternal dietary exposure to fiber during pregnancy and mammary tumorigenesis among rat offspring. Int J Cancer. 2006; 119(10):2279-86.

12. Bradley CS, Kennedy CM, Turcea AM, Rao SS, Nygaard IE..Constipation in pregnancy: prevalence, symptoms, and risk factors. Obstet Gynecol. 2007; 110(6):1351-7.

13. Ramakrishnan U, Grant FK, Imdad A, Bhutta ZA, Martorell R. Effect of Multiple Micronutrient versus Iron-Folate Supplementation during Pregnancy on Intrauterine Growth. Nestle Nutr Inst Workshop Ser. 2013; 74:53-62. 
14. Mudgil D, Barak S. Composition, properties and health benefits of indigestible carbohydrate polymers as dietary fiber: A review. Int J Biol Macromol. 2013; 61C:1-6. [Epub ahead of print]

15. Ley SH, Hanley AJ, Retnakaran R, Sermer M, Zinman B, O'Connor DL. Effect of macronutrient intake during the second trimester on glucose metabolism later in pregnancy. Am J ClinNutr. 2011; 94(5):1232-40.

16. Waldmann A, Ströhle A, Koschizke JW, Leitzmann C, Hahn A. Overall glycemic index and glycemic load of vegan diets in relation to plasma lipoproteins and triacylglycerols. Ann NutrMetab. 2007; 51(4):335-44. 